Tim Käseberg*, Jana Grundmann, Thomas Siefke, Stefanie Kroker und Bernd Bodermann

\title{
Abbildende Müller-Matrix-Ellipsometrie für die Charakterisierung vereinzelter Nanostrukturen
}

\author{
Imaging Mueller matrix ellipsometry for characterization of non-periodic nanostructures
}

https://doi.org/10.1515/teme-2021-0133

Eingang 15. Dezember 2021; angenommen 14. Februar 2022

Zusammenfassung: Ellipsometrie ist eine der vielseitigsten Methoden zur optischen Nanostrukturcharakterisierung. Insbesondere die Müller-Matrix-Ellipsometrie ermöglicht die Messung von optischen oder geometrischen Parametern mit Genauigkeiten bis in den Sub-NanometerBereich. In der konventionellen Ellipsometrie wird dabei über die komplette Beleuchtungspunktgröße gemittelt. Wenn der strukturierte Bereich auf der Probe kleiner ist als der Beleuchtungspunkt, oder die Struktur keine Periodizität aufweist, kann das Messergebnis durch in den Randbereichen reflektiertes Licht beeinträchtigt werden. Besonders problematisch ist dies bei freistehenden Nanostrukturen mit charakteristischen Größen kleiner als die Beleuchtungspunktgröße. In solchen Fällen kann abbildende Ellipsometrie genutzt werden. Dabei wird eine Müller-Matrix für jedes Pixel in einem Kamerabild gemessen, wodurch der Polarisationseinfluss der Probe lokal bestimmt wird. In diesem Beitrag liefern wir Ansätze, konkrete Zusammenhänge zwischen geometrischen Eigenschaften von Nanostrukturen auf Nebendiagonalelemente der Müller-Matrix zu ermitteln. Dazu haben wir einen Aufbau für die Messung von Müller-Matrix-Bildern bei verschiedenen Einfallswinkeln in Transmission und Reflexion realisiert sowie eine Probe gefertigt, mit der wir geometrische Struktureigenschaften in Müller-Matrix-Bildern systematisch messen. Wir stellen Messungen sowie numerische Simulationen zum Vergleich der Ergebnisse vor. Des Weiteren diskutieren wir thermische Einflüsse auf Mess-

\footnotetext{
*Korrespondenzautor: Tim Käseberg, Physikalisch-Technische Bundesanstalt Braunschweig, Bundesallee 100, 38116

Braunschweig, Deutschland, E-Mail: tim.kaeseberg@ptb.de, ORCID: https://orcid.org/0000-0003-1389-5117

Jana Grundmann, Bernd Bodermann, Physikalisch-Technische Bundesanstalt Braunschweig, Bundesallee 100, 38116 Braunschweig, Deutschland

Thomas Siefke, Friedrich-Schiller-Universität Jena, Institut für Angewandte Physik, Albert-Einstein-Straße 15, 07745 Jena, Deutschland

Stefanie Kroker, Technische Universität Braunschweig, Institut für Halbleitertechnik, LENA Laboratory for Emerging Nanometrology, Hans-Sommer-Str. 66, 38106 Braunschweig, Deutschland
}

ergebnisse und stellen einen Algorithmus zu deren Behandlung vor.

Schlagwörter: Nanometrologie, Ellipsometrie, MüllerEllipsometrie, Abbildende Ellipsometrie, Nanostrukturen.

Abstract: Ellipsometry is one of the most versatile methods for optical nanostructure characterization. Especially Mueller matrix ellipsometry allows the measurement of optical or geometrical parameters with uncertainties down to the sub-nanometer range. In conventional ellipsometry, this involves averaging over the complete illumination spot size. If the structured area on the sample is smaller than the illumination spot, or the structure has no periodicity, the measurement result may contain reflected signals from the surrounding areas. This is particularly noticeable for free-standing nanostructures with diameters smaller than the illumination spot size. In such cases, imaging ellipsometry can be used. Here, a Mueller matrix is measured for each pixel in a camera image, allowing the polarization influence of the sample to be investigated locally. In this paper, we provide approaches to determine concrete relationships between geometric properties of nanostructures on off-diagonal elements of the Mueller matrix. To this end, we have implemented a setup for measuring Mueller matrix images at different angles of incidence in transmission and reflection, and fabricated a sample with which we systematically measure geometric structural properties in Mueller matrix images. We present measurements as well as numerical simulations to compare the results. Furthermore, we discuss thermal influences on measurement results and present an algorithm for their treatment.

Keywords: Nanometrology, ellipsometry, Mueller ellipsometry, imaging ellipsometry, nanostructures.

\section{Einleitung}

Optische Messverfahren bieten viele Vorteile gegenüber höchstauflösenden Mikroskopieverfahren wie der Rasterkraftmikroskopie (atomic force microscopy, AFM) oder der Rasterelektronenmikroskopie (REM). Sie zeichnen sich 
nicht nur durch ihre Geschwindigkeit und Flexibilität, sondern auch durch ihren geringen Zerstörungsgrad aus. Eine der wichtigsten und vielseitigsten optischen Methoden im Bereich der Nanostrukturcharakterisierung ist die spektroskopische Ellipsometrie. Insbesondere die MüllerMatrix-Ellipsometrie, bei der alle Polarisationseigenschaften der untersuchten Probe in einer 4×4-Müller-Matrix zusammengefasst werden, ermöglicht die Messung von Schichtzusammensetzungen oder geometrischen Parametern periodischer Nanostrukturen mit Genauigkeiten bis in den Sub-Nanometer-Bereich [1, 2, 3, 4, 5]. In der konventionellen Ellipsometrie wird dabei stets ein Messergebnis erzielt, das über die komplette Größe des Beleuchtungspunkts gemittelt ist. Wenn der strukturierte Bereich auf der Probe allerdings kleiner ist als der Beleuchtungspunkt, oder wenn die zu untersuchende Struktur keine Periodizität aufweist, so kann das Messergebnis Einflüsse durch die zusätzlich ausgeleuchteten Randbereiche um die zu untersuchende Struktur herum enthalten. Besonders auffällig ist dies bei der Untersuchung von freistehenden Nanostrukturen mit Durchmessern viel kleiner als die Beleuchtungspunktgröße. Eine solche Messung ist praktisch ununterscheidbar von einer Messung am unstrukturierten Substrat allein.

In solchen Fällen, in denen die Struktur der Probe eine sinnvolle Untersuchung mit herkömmlicher Ellipsometrie erschwert, kann auf abbildende Ellipsometrie zurückgegriffen werden. Dabei handelt es sich um eine Verknüpfung aus Ellipsometrie und optischer Mikroskopie. Anstatt das von der Probe reflektierte oder transmittierte Licht nur $\mathrm{zu}$ detektieren, wird ein Abbildungssystem in den Strahlengang integriert und über eine Kamera ein Bild vom ausgeleuchteten Bereich der Probe aufgenommen. Auf diese Weise wird eine Müller-Matrix für jedes Pixel im Kamerabild gemessen, wodurch der Polarisationseinfluss der Probe lokal untersucht werden kann, unabhängig von der Größe des Beleuchtungspunkts. In der Auswertung können dann Bereiche mit unterschiedlichen Struktureigenschaften gesondert betrachtet werden, was insbesondere für die Defektcharakterisierung eine vielversprechende Anwendung darstellt $[6,7,8]$.

Die Auswertung von Müller-Matrix-Bildern geschieht dabei größtenteils nach den gleichen Algorithmen wie bei der herkömmlichen Ellipsometrie. Messergebnisse werden dazu über Bildausschnitte gemittelt, die homogene Bereiche der Probe enthalten, und dann mittels einfacher Modelle über die Lösung des inversen Problems die Strukturparameter in diesem Bereich ermittelt [9]. In diesem Beitrag liefern wir Ansätze, Müller-Matrix-Bilder stattdessen mit dreidimensionalen Modellen zu behandeln und konkrete Zusammenhänge zwischen messbaren
Effekten bestimmter Eigenschaften von Nanostrukturen auf Nebendiagonalelemente der Müller-Matrix zu ermitteln. Dazu haben wir einen Aufbau für die Messung von Müller-Matrix-Bildern bei verschiedenen Einfallswinkeln in Transmission und Reflexion umgesetzt sowie eine Probe gefertigt, mit der wir geometrische Struktureigenschaften in Müller-Matrix-Bildern systematisch messen. Messaufbau und Probe werden in Abschnitt 2 vorgestellt. Des Weiteren diskutieren wir in Abschnitt 3 die Auswirkungen von Stabilitäts-Einflüssen durch thermische Umgebungsbedingungen auf die Ergebnisse erster Messungen und stellen eine Software-Lösung für deren Behandlung vor. Anschließend werden in Abschnitt 4 Simulationsergebnisse besprochen und mit den Messungen verglichen. Abschnitt 5 bildet dann den Abschluss mit einer kurzen $\mathrm{Zu}$ sammenfassung des Beitrags.

\section{Methoden}

\subsection{Messaufbau}

Unser Messaufbau ist schematisch in Abbildung 1 als Draufsicht dargestellt. Der Aufbau besteht grundlegend aus zwei Armen: Der obere Arm (Beleuchtungsarm) beinhaltet die Komponenten der Beleuchtung und steht an einer festen Position. Der untere Arm (Analysearm) dient der Analyse des von der Probe reflektierten oder transmittierten Lichts unter verschiedenen Winkeln, weshalb er auf einem Rotationsversteller mit einem Innendurchmesser von $35 \mathrm{~cm}$ angebracht ist. Auf diese Weise lässt er sich um die Probe, die im Zentrum des Aufbaus auf mehreren Rotations- und Linearverstellern angebracht ist, rotieren, um so beliebige Einfalls- und Messwinkel sowie einen fließenden Übergang zwischen Reflexions- und Transmissionsmessungen realisieren zu können. Die möglichen Einfallswinkel werden dabei nur von der endlichen Größe der Objektive begrenzt und reichen für Reflexionsmessungen von $37,5^{\circ}$ bis $90^{\circ}[10]$.

Im Beleuchtungsarm dient eine blaue LED der Wellenlänge $455 \mathrm{~nm}$ als Lichtquelle. Das Licht der LED wird über mehrere Optiken gesammelt, kollimiert und für eine Köhler-Beleuchtung vorbereitet. Ein Teil des Lichts wird über einen 10:90-Strahlteiler an eine Photodiode als Intensitätsreferenz geleitet. Der größere Anteil wird durch einen Glan-Thompson-Polarisator und eine $\lambda / 4$-Platte geführt, die beide in Rotationsverstellern gelagert sind und die Justage der Polarisation der Beleuchtung ermöglichen. Anschließend wird eine Köhler-Beleuchtung realisiert, indem das Licht auf die Eintrittspupille eines Objektivs fo- 


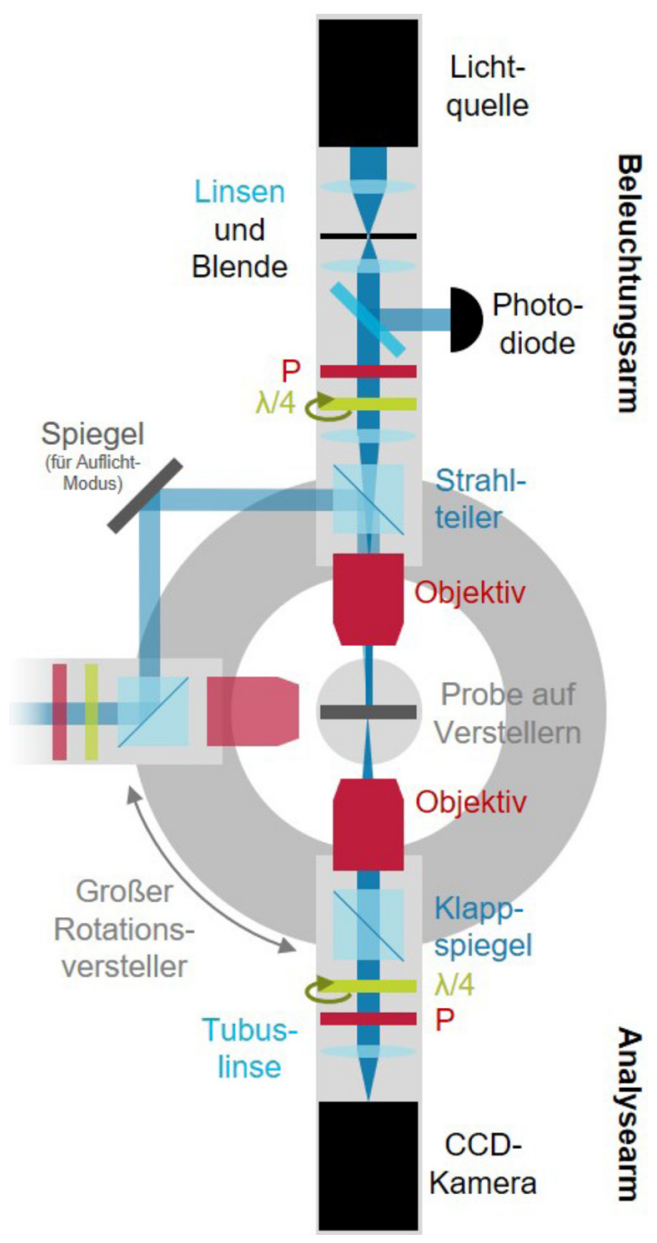

Abb. 1: Schematische Darstellung des abbildenden Müller-MatrixEllipsometer-Aufbaus. P: Polarisator, $\lambda / 4: \lambda / 4$-Platte.

kussiert wird [11, 12]. Die Probe wird dadurch möglichst homogen ausgeleuchtet. Bei abbildender Ellipsometrie limitieren die Abmaße des Beleuchtungspunktes nicht mehr die laterale Auflösung der Messung, da stattdessen über alle Pixel eines Kamerabildes gemessen wird. Daher ist eine gleichmäßige Ausleuchtung des Bildraumes hier von größerer Bedeutung als ein möglichst kleiner Beleuchtungspunkt.

Im Analysearm befindet sich wiederum ein Objektiv, das auf die Probe fokussiert ist. Die Objektive besitzen jeweils eine 50× Vergrößerung, eine numerische Apertur von 0,42 sowie einen Arbeitsabstand von $25,5 \mathrm{~mm}$, wodurch Probenstücke bis zu einem Durchmesser von etwa $5 \mathrm{~cm}$ kollisionsfrei bei allen zur Verfügung stehenden Einfallswinkeln vermessen werden können. Das vom Objektiv aufgenommene Licht wird zur Analyse der Polarisation durch eine weitere Kombination aus rotierbarer $\lambda / 4$-Platte und Polarisator geleitet und anschließend mit einer Tubuslinse auf eine monochromatische CCD-Kamera fokus- siert. Der Kamerachip besitzt $2688 \times 2200$ Pixel mit einer Pixelgröße von $(4,54 \mu \mathrm{m})^{2}$.

Neben einfachen Transmissions- und Reflexionsmessungen lassen sich mit dem Aufbau auch AuflichtMikroskopie-Messungen vornehmen. Dazu wird der Analysearm auf eine Position von $90^{\circ}$ zum Beleuchtungsarm gedreht, wie im transparenten Teil auf der linken Seite in Abbildung 1 skizziert. Das von der Probe zurück ins Objektiv des Beleuchtungsarms reflektierte Licht wird über einen 50:50-Strahlteiler zur Seite und mithilfe zweier Spiegel, die im Strahlengang platziert werden, in den Analysearm geleitet. Auf diese Weise können auch ohne weitere Umbaumaßnahmen Messungen bei senkrechtem Einfall durchgeführt werden.

Das Messprinzip des Aufbaus basiert auf einer horizontalen Realisierung einer sogenannten dual-rotating compensator Konfiguration. Bei dieser Form der Ellipsometrie wird die Beleuchtung über einen feststehenden Polarisator und eine rotierende Verzögerungsplatte (compensator) polarisiert und das von der Probe aufgenommene Licht wiederum über eine rotierende Verzögerungsplatte und einen feststehenden Polarisator analysiert. Während der Messung drehen sich die beiden $\lambda / 4$-Platten im Aufbau mit verschiedenen aber zueinander harmonischen Geschwindigkeiten, wodurch eine Modulation der detektierten Intensität $I$ erreicht wird, die neben den Polarisationsoptiken auch von den Elementen der zu messenden Müller-Matrix der Probe abhängt:

$$
I=c \cdot \sum_{i, j=1}^{4} a_{i} p_{j} m_{i j}
$$

Hierbei sind $p$ und $a$ die Elemente der Stokesvektoren, die den Einfluss der beiden Ellipsometer-Arme auf unpolarisiertes Licht beschreiben, $c$ ist eine Konstante für die Empfindlichkeit der verwendeten Kamera und $m_{i j}$ sind die Elemente der Müller-Matrix der Probe:

$$
\underline{M}=\left(\begin{array}{llll}
m_{11} & m_{12} & m_{13} & m_{14} \\
m_{21} & m_{22} & m_{23} & m_{24} \\
m_{31} & m_{32} & m_{33} & m_{34} \\
m_{41} & m_{42} & m_{43} & m_{44}
\end{array}\right) .
$$

Aus der modulierten Intensität lassen sich dann über eine Fourier-Analyse für jedes Pixel der Kamera die MüllerMatrix-Elemente $m_{i j}$ gewinnen, die zur weiteren Analyse zu 16 sogenannten Müller-Matrix-Bildern zusammengesetzt werden [13, 14]. 


\subsection{Probe}

Um den Einfluss bestimmter Probengeometrien auf die einzelnen Müller-Matrix-Elemente näher zu untersuchen, haben wir eine Reihe von vereinzelten Nanostrukturen mit variierenden Parametern gefertigt. Bei den Strukturen handelt es sich um Vertiefungen in einer $100 \mathrm{~nm}$ dicken Schicht Polymethylmethacrylat (PMMA) auf einem Silizium-Substrat. Abbildung 2 zeigt eine Übersicht über die Probe. Die Strukturen sind in einem Raster im Abstand von $5 \mathrm{~mm}$ zueinander angeordnet, um Interaktionen zwischen verschiedenen Strukturen auszuschließen. Die Parameter der Strukturen in den Zeilen A bis D variieren dabei über die Spalten 1 bis 10, während die Zeile E als Substratreferenz freigelassen wurde. In Zeile A befinden sich $(5 \mu \mathrm{m})^{2}$ große Quadratstrukturen mit abgerundeten Ecken. Die Eckenradien variieren über die Spalten von $100 \mathrm{~nm}$ bis $2 \mu \mathrm{m}$. Damit wird ein Übergang von annähernd quadratisch zu annähernd kreisförmig realisiert. Die Zeilen $B$ und $C$ enthalten jeweils quadratische und kreisförmige Strukturen mit Durchmessern von $50 \mathrm{~nm}$ bis $2 \mu \mathrm{m}$, sodass Messungen auch an Strukturen bis weit unterhalb der Auflösungsgrenze des abbildenden Systems durchgeführt werden können. Zeile D enthält kleine Gitter mit bis $\mathrm{zu} 4 \times 4$ quadratischen, $1 \mu \mathrm{m}$ großen Strukturen, mit $10 \mu \mathrm{m}$ und $20 \mu \mathrm{m}$ großen Abständen. Die kleinen Gitter erlauben es, Untersuchungen zu Einflüssen geringer Periodizität auf die Elemente der Müller-Matrix umzusetzen [15].

\section{Messungen}

\subsection{Gemessene Müller-Matrix-Bilder}

Es wurden zunächst Messungen der Müller-Matrix-Bilder an den Strukturen der Zeile A durchgeführt. Als Beispiel finden sich Messergebnisse der Struktur A5 mit einem Eckenradius von $400 \mathrm{~nm}$ in Abbildung 3. Messungen von Müller-Matrix-Bildern werden in Anlehnung an gewöhnliche Müller-Matrizen in einer 4×4-Anordnung dargestellt, wobei jedes Einzelbild die Ergebnisse aller Pixel für ein Müller-Matrix-Element zusammenfasst. Die Darstellung der Müller-Matrix-Werte erfolgt in diesem Beitrag farbcodiert. Da das erste Matrix-Element $m_{11}$ größtenteils nur die Intensität der Messung widerspiegelt, werden alle anderen Matrix-Elemente wie üblich auf dieses Element normiert. Die Messungen wurden im Auflicht-Modus des Aufbaus durchgeführt, also bei einem Einfallswinkel von $0^{\circ}$. Trotz einer faktischen Reflexionsmessung weisen die gemessenen Matrix-Elemente zum Teil Eigenschaften einer Transmissionsmessung auf. Am deutlichsten wird dies

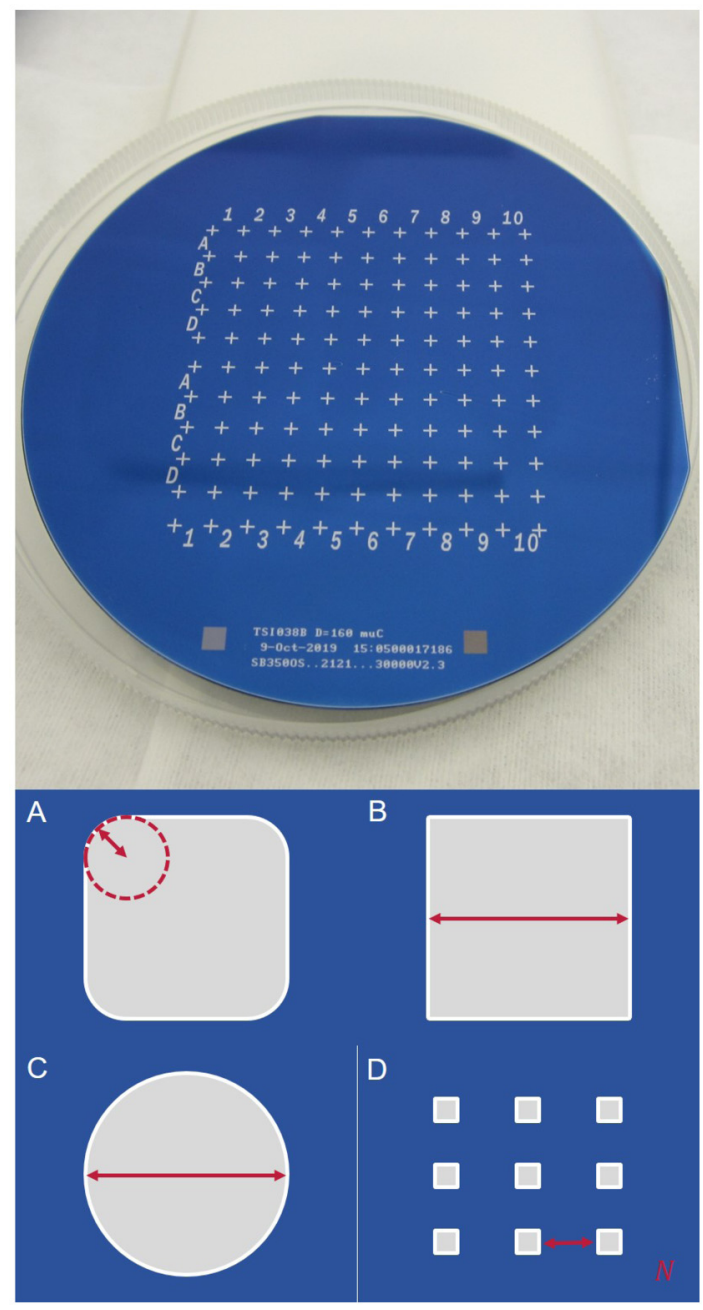

Abb. 2: Übersicht über den Probenwafer und die Strukturen in den Zeilen A bis D.

an den durchweg positiven Elementen der Hauptdiagonale. Grund hierfür ist das Umlenken des Lichts über mehrere Spiegel auf dem Weg zum Analysearm während einer Auflichtmessung. Die Reflexionen drehen Teile der MüllerMatrix, was zu Vorzeichenunterschieden führt, sodass sie am Ende charakterlich wie die Matrix einer Transmissionsmessung wirkt. Des Weiteren wurden die Bilder auf Referenzmessungen von unstrukturiertem Substrat normiert, um Einflüsse sowohl des Substrats selbst als auch der im Strahlengang befindlichen Optiken, der Objektive und Spiegel, zu eliminieren [10]. Nichtsdestotrotz zeigen manche der Nebendiagonalelemente einen leichten Offset, der auf geringe Abweichungen in der Justage der Polarisationsoptiken hindeutet. Darüber hinaus sind in den Messungen deutliche Einflüsse der Strukturkanten zu beobachten, teilweise mit unterschiedlichem Vorzeichen für Kanten verschiedener Orientierung. Zugleich ist be- 


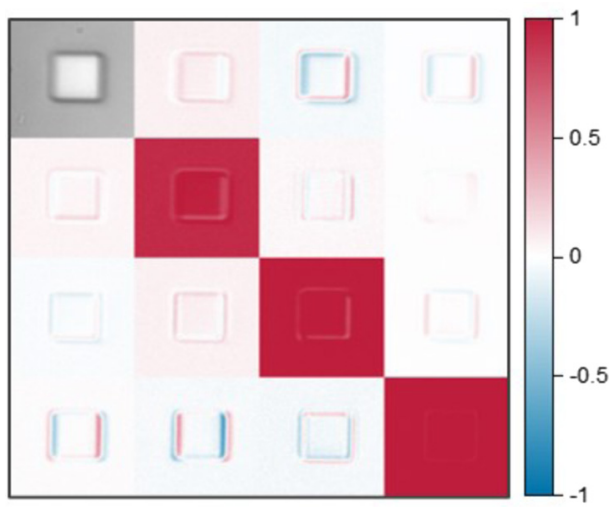

Abb. 3: Gemessene Müller-Matrix-Bilder der Struktur A5 mit Eckenradius $400 \mathrm{~nm}$. Effekte der Randbereiche der Struktur auf die lokale Müller-Matrix sind zu erkennen, werden jedoch durch thermischen Drift beeinflusst.

sonders in $m_{11}$ eine Art Verschmierung zu erkennen. Diese Verschmierung scheint sich ebenso auf die Ergebnisse in den anderen Müller-Matrix-Bildern auszuwirken. Grund dieser Verschmierung ist mit großer Wahrscheinlichkeit ein thermischer Drift der Struktur im Bild. Während einer Messung werden sukkzessive Einzelbilder bei verschiedenen Stellungen der beiden Verzögerungsplatten aufgenommen. Aus dieser Reihe an Bildern werden im anschließenden Auswerteverfahren die Müller-Matrix-Bilder ermittelt. Die hier vorliegenden Messergebnisse benötigten für diese schrittweise Messung jeweils etwa sieben Minuten pro vollständigem Messdurchlauf. Thermische Fluktuationen im Laborklima, zum Beispiel durch das Verlassen des Raumes nach Start einer Messreihe, können zu Verschiebungen im Aufbau führen, die dafür sorgen, dass die Probe im Bild wandert. Abbildung 4 zeigt dazu den Weg einer wandernden Struktur sowie die Raumtemperatur in Probennähe. Dazu wurden über einen Zeitraum von 8 Stunden in Abständen von 10 Sekunden Bilder einer einjustierten Struktur aufgenommen und anschließend deren Position im Bild lokalisiert. Die Messung wurde nachts durchgeführt, um Störeinflüsse innerhalb des Gebäudes möglichst zu reduzieren. Auch elektromagnetische und tektonische Einflüsse auf die Messung können aufgrund der Lage und Bauart des Labors zunächst vernachlässigt werden. Der Verlauf in Abbildung 4 zeigt eine leicht verzögerte Antwort der Strukturposition auf die aktuelle Temperatur im Raum. Selbst nach mehreren Stunden wandert die Probe noch mit ungefähr $100 \mathrm{~nm}$ pro Minute, was in einer für die durchgeführten Messreihen signifikanten Größenordnung liegt.

Abgesehen von den Strukturen in Zeile A wurden erste Messungen an den Strukturen der Zeile $\mathrm{C}$ als Test über

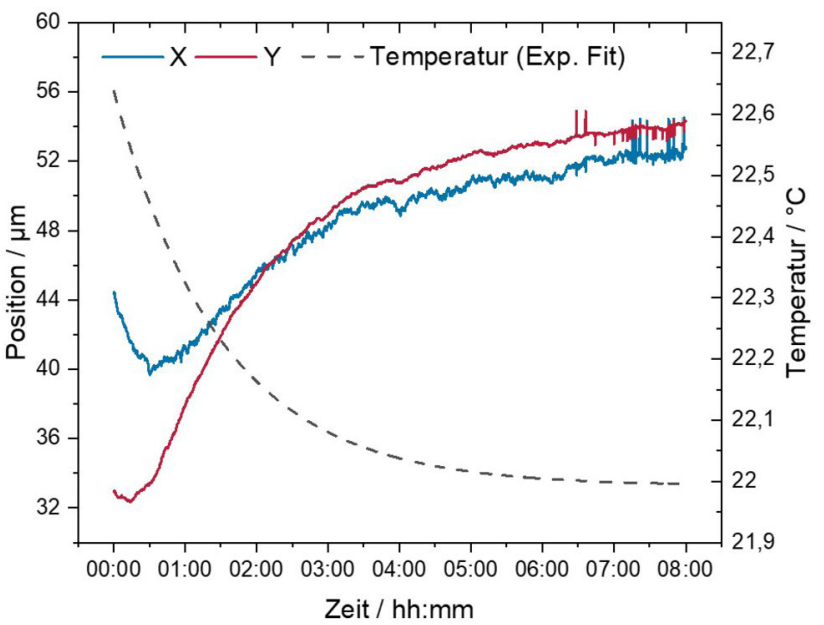

Abb. 4: Position einer Struktur und Raumtemperatur gemessen über 8 Stunden. Während der Messreihe wurden Bilder aufgenommen, aus denen die Strukturposition ermittelt wurde.

das Auflösungsvermögen des implementierten abbildenden Systems durchgeführt. Allerdings erwies sich hier der thermische Drift im Verhältnis zur Strukturgröße als zu ausschlaggebend um verlässliche Aussagen zu erhalten.

\subsection{Drift-Korrektur-Algorithmus}

Während der Messung der Einzelbilder wanderte die Struktur innerhalb des Bildes um wenige Mikrometer. Da für die Berechnung der Müller-Matrix-Bilder aber die Informationen der jeweiligen Pixelkoordinaten aller Bilder der Messreihe miteinander verrechnet werden, entsteht durch diesen Drift eine Verschmierung der Messergebnisse. Vor einer notwendigen thermischen Stabilisierung des Messsystems haben wir daher einen Korrektur-Algorithmus in das Auswerteverfahren implementiert, um diesen Verschmierungen entgegenzuwirken.

Ausgangspunkt für die Drift-Korrektur ist immer das erste Bild der Messreihe. Um die Rechenzeiten zu minimieren wird zu Beginn ein Bereich von 400×400 Pixeln um die zu zentrierende Struktur manuell ausgewählt. Dabei ist es noch nicht von Bedeutung, wo genau die Struktur innerhalb dieses Bereichs liegt. Das aufgenommene Bild wird dann zunächst auf ein Referenzbild ohne Struktur normiert und ein Histogramm der Grauwerte des Bildes erstellt. Auf das Bild der Struktur werden nun Sobel-Operatoren angewandt. Dabei handelt es sich um Kantendetektions-Operatoren, die hohe lokale Intensitätsunterschiede in Form von Gradienten visualisieren [16]. Um den Gradienten eines Pixels zu berechnen, werden dazu das entsprechende Pixel sowie seine direkten Nach- 
barn in Form eines 3×3-Pixel-Bildausschnitts betrachtet und mit den Sobel-Operatoren

$$
S_{x}=\left(\begin{array}{ccc}
-1 & 0 & 1 \\
-2 & 0 & 2 \\
-1 & 0 & 1
\end{array}\right) \text { und } S_{y}=\left(\begin{array}{ccc}
1 & 2 & 1 \\
0 & 0 & 0 \\
-1 & -2 & -1
\end{array}\right)
$$

gefaltet. Die Ergebnisse dieser Faltung sind wiederum $3 \times 3$-Matrizen, die aufsummiert und miteinander kombiniert werden, um den neuen Grauwert zu erhalten, der den Gradienten an dem entsprechenden Pixel wiedergibt. Das entstandende neue Grauwert-Bild wird nun wie eine Höhenkarte behandelt und eine sogenannte Wasserscheidentransformation darauf angewandt, um Segmente innerhalb des Bildes zu finden. Bei der Wasserscheidentransformation werden zusammenhängende Bereiche eines Bildes so befüllt als wären es Vertiefungen einer Landmasse, die mit Wasser geflutet werden [17]. Aus dem Histogramm des ursprünglichen Bildes werden dazu Extremstellen herausgesucht, um Punkte im Gradientenbild zu finden, die sich als Marker eignen. Von diesen Markern aus wird dann begonnen, das Gradientenbild zu fluten, allerdings ohne dass Bereiche ausgehend von unterschiedlichen Markern sich zusammenfügen. Auf diese Weise wird ein zusammenhängender Bereich gefunden, der die Struktur im Bild abdeckt. Abschließend wird der Mittelpunkt dieses ermittelten Bereichs in Form eines Massenschwerpunkts berechnet. Dieser Massenschwerpunkt stellt das Zentrum der detektierten Struktur im Bild dar. Der aktuelle Bildausschnitt der Messreihe wird dann auf diesen Massenschwerpunkt neu zentriert. Zudem werden die Koordinaten des neuen Zentrums an die nächste Iteration weitergegeben und als Mittelpunkt des 400×400-PixelBildausschnitts des nächsten Bildes der Messreihe verwendet. Auf diese Weise wird die gesamte Messreihe durchlaufen und aus jedem Bild ein Bildausschnitt gewonnen, in dem die Struktur zentriert vorliegt. Zum Schluss wird dann die Evaluation der Müller-Matrix-Bilder aus diesen zentierten Bildausschnitten vorgenommen. Die DriftKorrektur wurde erfolgreich auf alle Messungen der Strukturen in Zeile A angewandt. Für die Messungen in Zeile $\mathrm{C}$ konnte allerdings aufgrund der niedrigen Strukturgröße die Wasserscheidentransformation nicht immer verlässlich durchgeführt werden, sodass eine Neuzentrierung der Bilder hier nicht möglich war. Abbildung 5 zeigt die mit der Drift-Korrektur behandelten Müller-Matrix-Bilder am Beispiel der Struktur A5. Im direkten Vergleich mit Abbildung 3 zeigt sich eine deutliche Abnahme der Verschmierungen in den Bildern. Die gleichzeitige Reduktion mancher Müller-Matrix-Werte deutet darauf hin, dass manche der beobachteten Effekte teilweise durch den Drift

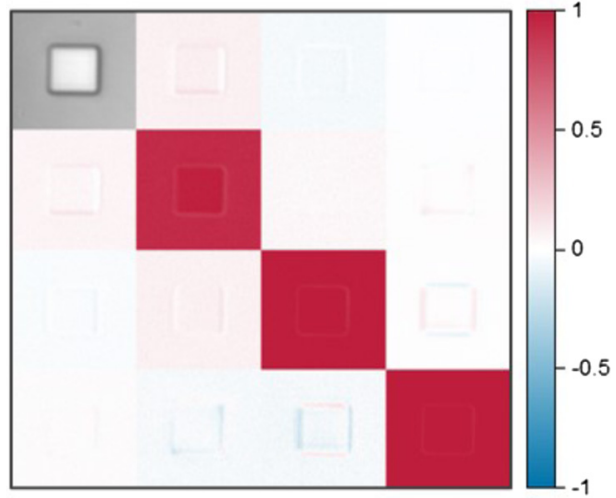

Abb. 5: Drift-korrigierte Müller-Matrix-Bilder der Struktur A5. Die Bilder zeigen nun lokale Einflüsse der Struktur auf die Müller-Matrix ohne Drift-Effekte.

der Struktur hervorgerufen wurden. Um zu bestimmen, welche der Effekte tatsächliche lokale Einflüsse der Struktur sind und ob eine solche Drift-Korrektur ausreicht, um sie hervorzuheben, werden im nächsten Schritt numerische Simulationen hinzugezogen.

\section{Numerische Simulationen}

\subsection{Durchführung der Simulationen}

Für die Auswertung von ellipsometrischen Messungen werden in der Regel Simulationen durchgeführt, um das inverse Problem zu lösen und aus den Messergebnissen Strukturparameter wie Schichtdicken, Perioden oder optische Konstanten zu gewinnen. Für einfache unstrukturierte Proben werden dazu sowohl in der konventionellen als auch in der abbildenden Ellipsometrie Modelle von Schichtsystemen herangezogen, während bei periodischen Nanostrukturen auf zweidimensionale QuerschnittModelle der Struktur zurückgegriffen wird. Für die Simulation von nicht-periodischen Strukturen wie den von uns verwendeten Proben ist es jedoch erforderlich, dreidimensionale Modelle der Strukturen zu erstellen, um den lokalen Einfluss der geometrischen Parameter von beispielsweise abgerundeten Ecken auf die Polarisation vollständig rekonstruieren zu können. Anders als Modelle mit geringerer Dimensionalität sind dreidimensionale Simulationen wesentlich komplexer und benötigen deutlich mehr Rechenressourcen in Form von Zeit und Arbeitsspeicher, was durch die Größe mancher Strukturen noch beeinträchtigt wird. Abgesehen davon muss für eine korrekte Rekonstruktion der gemessenen Müller-Matrix-Bilder die Beleuchtung bei der Simulation der Mikroskop-Bilder mög- 
lichst realistisch sein. Dies beinhaltet die Überlagerung mehrerer ebener Wellen aus unterschiedlichen Richtungen, also mit unterschiedlichen Pupillenpunkten innerhalb der numerischen Apertur im Beleuchtungsstrahlengang. Für eine realistische Darstellung ist eine hohe Anzahl von simulierten Pupillenpunkten genauso ausschlaggebend wie deren sinnvolle Verteilung innerhalb der numerischen Apertur, wobei beide Faktoren die Rechenzeit signifikant beeinflussen. Die numerischen Simulationen für diese Arbeit wurden mithilfe der Finite-ElementeMethode und des Programms JCMsuite durchgeführt [18]. Aus den eben genannten Gründen wurden die Simulationen beschränkt auf die Zielparameter aus dem Strukturdesign. Aus AFM-Messungen der gefertigten Strukturen ist bekannt, dass deren Größen zu 2,1\% und Eckenradien $\mathrm{zu} 6,8 \%$ an die Zielparameter heranreichen und diese Annahme daher gerechtfertigt ist. Des Weiteren wurden die Simulationen vorerst nur mit einem einzigen Pupillenpunkt bei senkrechter Inzidenz simuliert. Unter diesen Voraussetzungen dauerte die Simulation einer einzelnen Struktur etwa 2 Stunden und benötigte ungefähr 350 GB RAM.

\subsection{Simulationsergebnisse}

Die Ergebnisse der numerischen Simulationen sind beispielhaft für die Struktur A5 in Abbildung 6 dargestellt. Da die Simulationen echte Auflichtmessungen ohne weitere Spiegelungen darstellen sind die Vorzeichen mancher Matrix-Elemente im Vergleich zu den Messungen verdreht. Die in den simulierten Müller-Matrix-Bildern sichtbaren Effekte weisen zu den aus den Drift-korrigierten gemessenen Bildern eine signifikante Ähnlichkeit auf. Dies deutet darauf hin, dass die Drift-Korrektur nur die Einflüsse des thermischen Drifts entfernt hat und die noch verbleibenden messbaren Effekte Einflüsse der lokalen Strukurgeometrie auf die Müller-Matrix sind. Diese Effekte bilden zusätzliche Informationen, die bei der Formcharakterisierung von Nanostrukturen eingesetzt werden können und die über den Informationsgehalt optischer Mikroskopie allein hinausgehen. Vergleicht man beispielsweise in Abbildung 7 die Bilder der $m_{11}$-Elemente für die Strukturen $\mathrm{A} 1$ und $\mathrm{A} 5$, so erkennt man zunächst keinen nennenswerten Unterschied, obwohl sich die Eckenradien beider Strukturen um $300 \mathrm{~nm}$ unterscheiden. Deutliche Unterschiede zwischen beiden Messungen sind jedoch in anderen Müller-Matrix-Elementen zu erkennen. So ist zum Beispiel in $m_{24}$ und $m_{42}$, aber auch in $m_{31}$ und $m_{13}$ der Sprung von $100 \mathrm{~nm}$ auf $400 \mathrm{~nm}$ Eckenradius deutich zu beobachten. Betrachtet man das $m_{24}$-Element in Abbildung 7

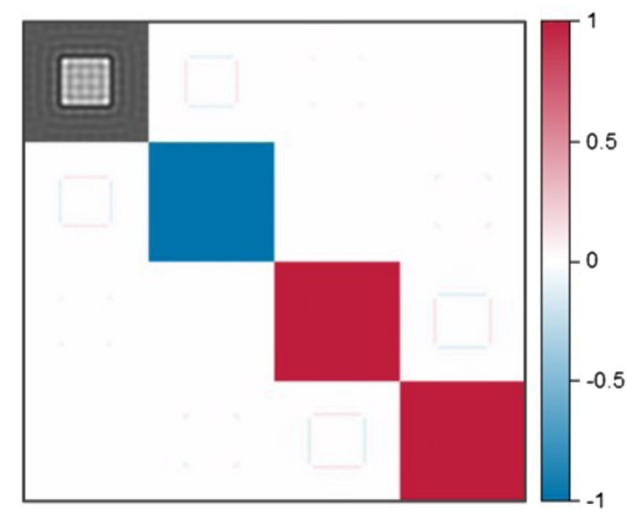

Abb. 6: Simulierte Müller-Matrix-Bilder der Struktur A5. Die Effekte sind bis auf umgekehrte Vorzeichen vergleichbar mit denen der korrigierten Messung.

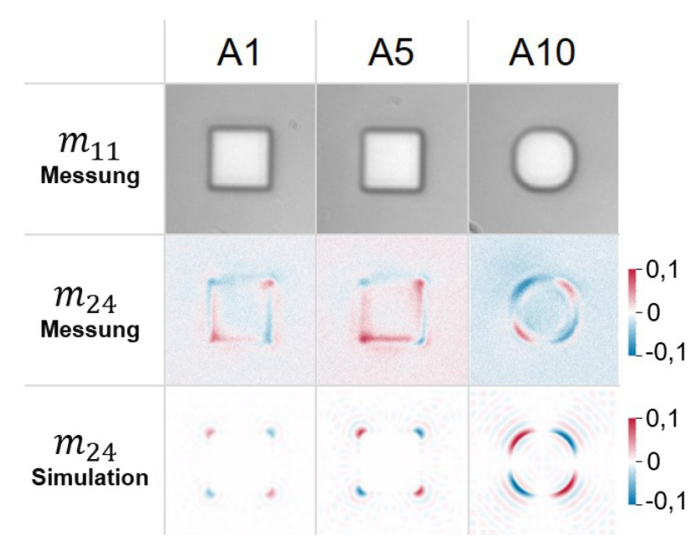

Abb. 7: Vergleich der Elemente $m_{11}$ und $m_{24}$ aus korrigierter Messung und Simulation für die Strukturen A1, A5 und A10. Die in den Ecken der Struktur beobachtbaren Effekte weisen in Messung und in Simulation ähnliche Abhängigkeiten vom Radius der Ecke auf.

allein, so zeigt sich in den Simulationen ein Anstieg der Absolutwerte der Müller-Matrix in den Ecken der Nanostruktur um $85 \%$ von A1 zu A5 und um $163 \%$ von A5 zu A10. Der Unterschied in den Messergebnissen fiel mit 15\% von A1 zu A5 und $166 \%$ von A5 zu A10 ähnlich signifikant aus. Abweichungen zur Simulation lassen sich damit begründen, dass die Simulationsergebnisse Idealbedingungen darstellen, in denen noch nicht alle Einflüsse der Beleuchtung oder des Pixelrauschens komplett berücksichtigt wurden.

\section{Zusammenfassung}

In diesem Beitrag stellten wir unseren Aufbau für die abbildende Müller-Matrix-Ellipsometrie vor, den wir an 
speziell entworfenen Nanostrukturen getestet haben, um den Zusammenhang zwischen geometrischen Merkmalen und Nebendiagonalelementen der Müller-Matrix weiter zu untersuchen. Unser Aufbau ermöglicht es, sowohl Transmissions- als auch Reflexionsmessungen bei beliebigen Winkelkombinationen im sichtbaren Wellenlängenbereich durchzuführen. Reflexionsmessungen können dabei zwischen $37,5^{\circ}$ und $90^{\circ}$ Einfallswinkel durchgeführt werden. Außerdem verfügt der Aufbau über einen AuflichtMikroskopiemodus, der Reflexionsmessungen bei senkrechtem Einfall ohne größere Umbauten ermöglicht. Um den Einfluss der Form der Nanostrukturen auf die MüllerMatrix-Elemente $z u$ untersuchen, haben wir eine Reihe von Strukturen mit einfachen geometrischen Formen und Größen zwischen $50 \mathrm{~nm}$ und $5 \mu \mathrm{m}$ entworfen und hergestellt. Wir haben quadratische Strukturen mit verschiedenen Eckenradien sowie kreisförmige Strukturen mit unserem Aufbau gemessen. Aufgrund von thermischen Drifts der Probe konnten die kleineren kreisförmigen Strukturen nicht sinnvoll ausgewertet werden. Zum Ausgleich des thermischen Drifts wurde ein Korrektur-Algorithmus implementiert und vorgestellt. Für die größeren quadratischen Strukturen mit Eckenrundung wurde erfolgreich der Algorithmus zur Driftkorrektur angewandt und die resultierenden korrigierten Müller-Matrix-Bilder mit FEMSimulationen der Designstrukturen verglichen. Auch ohne eine Anpassung der Simulationen an die Messungen konnten wir eine große Ähnlichkeit zwischen den Messungen und den Simulationen feststellen. Darüber hinaus zeigten sowohl die Messungen als auch die Simulationen einen signifikanten Einfluss einer Änderung des Eckenradius der Strukturen von $100 \mathrm{~nm}$ auf $400 \mathrm{~nm}$ auf die Nebendiagonalelemente der Müller-Matrix, vor allem $m_{24}$ und $m_{42}$. Dies unterstreicht das Potenzial der abbildenden Müller-Matrix-Ellipsometrie für messtechnische Anwendungen zur Charakterisierung von nicht-periodischen Nanostrukturen im Sub-Wellenlängenbereich. Die genaue Analyse von Nanoform-Probeneigenschaften aus einzelnen Elementen der Müller-Matrix ist Thema weitergehender Untersuchungen und wird in Folgepublikationen detaillierter diskutiert.

Finanzierung: This work is funded through the project 17FUN01 (European Association of National Metrology Institutes) "BeCOMe" within the Programme EMPIR. The EMPIR initiative is co-founded by the European Union's Horizon 2020 research and innovation program and the EMPIR Participating Countries.

This project (20FUN02 "POLight”, European Association of National Metrology Institutes) has received funding from the EMPIR programme co-financed by the Participating States and from the European Union's Horizon 2020 research and innovation programme.

S. K. also acknowledges partial support by the Deutsche Forschungsgemeinschaft (DFG, German Research Foundation) under Germanys Excellence Strategy - EXC2123 QuantumFrontiers - 390837967.

\section{Literatur}

1. A. Rothen, The ellipsometer, an apparatus to measure thicknesses of thin surface films, Rev. Sci. Instrum. 16 (1945), 26-30.

2. K. Vedam, Spectroscopic ellipsometry: a historical overview, Thin Solid Films 313-314 (1998), 1-9.

3. M. Losurdo, Applications of elipsometry in nanoscale science: Needs, status, achievements and future challenges, Thin Solid Films 519 (2011), 2575-2583.

4. Y. Tan, C. Chen, X. Chen, W. Du, H. Gu und S. Liu, Development of a tomographic Mueller-matrix scatterometer for nanostructure metrology, Rev. Sci. Instrum. 89 (2018), 073702.

5. I. Voloshenko, B. Gompf, A. Berrier, M. Dressel, G. Schnoering, M. Rommel und J. Weis, Mueller matrix metrology: Depolariztion reveals size distribution, Appl. Phys. Lett. 115 (2019), 063106.

6. N. Kerwien, Zum Einfluss von Polarisationseffekten in der mikroskopischen Bildentstehung, Doktorarbeit, Universität Stuttgart, 2007.

7. S. Liu, W. Du, X. Chen, H. Jiang und C. Zhang, Mueller matrix imaging ellipsometry for nanostructure metrology, Opt. Express 23 (2015), 17316-17329.

8. S. Chandel, J. Soni, S. K. Ray, A. Das, A. Ghosh, S. Raj und N. Ghosh, Complete polarization characterization of single plasmonic nanoparticle enabled by a novel Dark-field Mueller matrix spectroscopy system, Sci. Rep. 6 (2016), 26466.

9. C. Wang, X. Chen, C. Chen, S. Sheng, L. Song, H. Gu, H. Jiang, C. Zhang und S. Liu, Reconstruction of finite deep sub-wavelength nanostructures by Mueller-matrix scattered-field microscopy, Opt. Express 29 (2021), 32158.

10. J. Grundmann, Konzeption und Realisierung eines experimentellen Müller-Matrix-Mikroskop-Aufbaus, Masterarbeit, Technische Universität Braunschweig, 2020.

11. A. Köhler, Ein neues Beleuchtungsverfahren für mikrophotographische Zwecke, Zeitschrift für wissenschaftliche Mikroskopie und für Mikroskopische Technik 10 (1893), 433-440.

12. H. Gross, Handbook of Optical Systems, Volume 1, Fundamentals of Technical Optics, Wiley-Vch, 2005.

13. R. Azzam, Photopolarimetric measurement of the Mueller matrix by Fourier analysis of a single detected signal, Opt. Lett. 2 (1978), 148-150.

14. D. Goldstein, Polarized Light, CRC Press, 2017.

15. T. Käseberg, J. Dickmann, T. Siefke, M. Wurm, S. Kroker und B. Bodermann, Mueller matrix ellipsometry for enhanced optical form metrology of sub-lambda structures, in: Modeling Aspects in Optical Metrology VII 11057, SPIE, München, 2019, 155-165. 
16. P. E. Danielsson und O. Seger, Generalized and Separable Sobel Operators, in: Machine Vision for Three-Dimensional Scenes, Freeman, Academic Press, Boston, 1990.

17. S. Beucher und F. Meyer, Segmentation: The watershed transformation. Mathematical morphology in image processing, Opt. Eng. 34 (1993), 433-481.

18. JCMsuite. The Simulation Suite for Nano-Optics. JCMwave $\mathrm{GmbH}$, Berlin, https://www.jcmwave.com/, Letzter Zugriff: 29. Oktober 2021.

\section{Autoreninformationen}

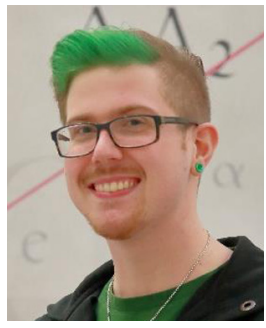

\section{Tim Käseberg}

Physikalisch-Technische Bundesanstalt Braunschweig, Bundesallee 100, 38116

Braunschweig, Deutschland

tim.kaeseberg@ptb.de

Tim Käseberg studierte Physik an der Technischen Universität Braunschweig und ist seit 2018 Doktorand der Arbeitsgruppen „Optische Nanometrologie“ und „Metrologie für funktionale Nanosysteme“ der Physikalisch-Technischen Bundesanstalt Braunschweig. Zu seinen Forschungsinteressen gehören optische Verfahren zur Untersuchung nanoskaliger Strukturen, insbesondere die MüllerMatrix-Ellipsometrie.

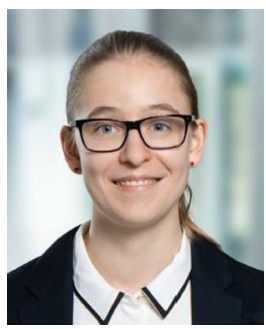

\section{Jana Grundmann}

Physikalisch-Technische Bundesanstalt Braunschweig, Bundesallee 100, 38116 Braunschweig, Deutschland jana.grundmann@ptb.de

Jana Grundmann studierte an der Technischen Universität Braunschweig Elektrotechnik und ist seit 2021 Doktorandin an der Physikalisch-Technischen Bundesanstalt in der Arbeitsgruppe „Optische Nanometrologie“. Ihr Arbeitsgebiet ist die abbildende MüllerMatrix-Ellipsometrie in Zusammenhang mit der Charakterisierung von nanoskaligen Strukturen und Defekten.

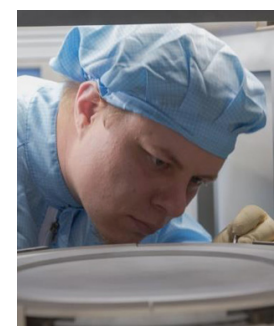

\section{Dr. Thomas Siefke}

Friedrich-Schiller-Universität Jena, Institut für Angewandte Physik,

Albert-Einstein-Straße 15, 07745 Jena,

Deutschland

thomas.siefke@uni-jena.de

Thomas Siefke absolvierte den Studiengang „scientific instrumentation“ mit dem Schwerpunkt Mikro- und Nanotechnologien an der Ernst-Abbe-Hochschule in Jena. Die nachfolgende Promotion zum Thema „Entwicklung von Drahtgitterpolarisatoren für Anwendungen im ultravioletten bis in den vakuumultravioletten Spektralbereich“ erfolgte an der Friedrich-Schiller-Universität Jena in Kooperation mit der Physikalisch-Technischen Bundesanstalt in Braunschweig. Aktuell koordiniert er als Postdoc die Mikro- und Nanostrukturierung am IAP-Jena.

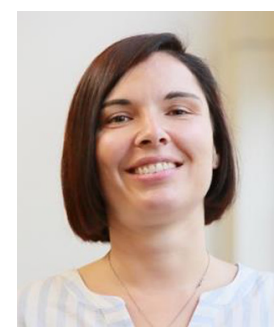

Prof. Dr. Stefanie Kroker

Technische Universität Braunschweig, Institut für Halbleitertechnik, LENA Laboratory for Emerging Nanometrology, Hans-Sommer-Str. 66, 38106 Braunschweig, Deutschland s.kroker@tu-braunschweig.de

Stefanie Kroker absolvierte ein Physik-Studium an der FriedrichSchiller-Universität Jena und der Universidad de Granada/Spanien und ist seit 2016 Professorin an der Technischen Universität Braunschweig, wo sie seit 2021 die Abteilung „Hybride Integration für die optische Quantenmetrologie“ leitet. Ihre Forschungsinteressen umfassen mikro- und nanophotonische Systeme für die optische Hochpräzisions- und Quantenmetrologie sowie deren Charakterisierung.

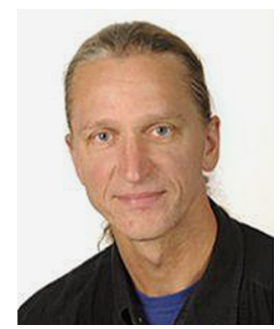

\section{Dr. Bernd Bodermann}

Physikalisch-Technische Bundesanstalt Braunschweig, Bundesallee 100, 38116 Braunschweig, Deutschland bernd.bodermann@ptb.de

Bernd Bodermann studierte Physik und promovierte 1998 an der Universität Hannover, Institut für Quantenoptik. Seit 1999 arbeitet er an der Physikalisch-Technischen Bundesanstalt auf dem Gebiet der dimensionellen Messtechnik und ist seit 2006 Leiter der Arbeitsgruppe „Optische Nanometrologie“. Seine Forschungsinteressen umfassen quantitative Mikroskopie, Scatterometrie, Ellipsometrie und rigorose optische Modellierung für Anwendungen z. B. in der Halbleiterfertigung, Nanotechnologie, Metamaterialien und diffraktiver Optik. 